\title{
Ultrasensitive Label-Free Microcavity Biosensors with High Selectivity
}

\author{
Erol Ozgur, Mehmet Bayindir \\ UNAM-Institute of Materials Science and Nanotechnology \\ Bilkent University \\ Ankara, Turkey \\ ozgur@nano.org.tr
}

\author{
Ozan Aktas, Mehmet Bayindir \\ Department of Physics \\ Bilkent University \\ Ankara, Turkey
}

\begin{abstract}
High quality factor whispering gallery mode microresonators have been recently shown to exhibit detection sensitivity of single molecule; however, the selectivity of these sensors among different types of analytes remains as an important issue, obscuring the broad applicability of optical microcavities. We demonstrate a surface modification strategy for fabrication of high selectivity and sensitivity microcavity biosensors in this study.
\end{abstract}

Microtoroid, whispering gallery mode, self assembly monolayer

\section{INTRODUCTION}

Microresonators, especially when equipped with high quality factors could be utilized as sensors having extremely high sensitivity. Especially for biological sensing applications, microresonators provide incredible opportunities, including single molecule sensing. There are several important examples of biological sensors developed by utilization of the shift of the WGM of microresonators. Microspheres were shown to be used for detection of various biological species including DNA, proteins and viruses (1). Microtoroid sensors were also shown to detect single protein binding events (2).

Specificity is definitely as critical as sensitivity when a sensor is considered, since lack of differentiation of the target from any other species, namely false positives, could be as misleading as false negative results. Although high sensitivity is an accomplished issue, there are a limited number of examples regarding high selectivity in literature (3), and single entity level detection with high selectivity have not been demonstrated yet, to our knowledge. In this work, we propose and demonstrate a method to attain highly selective microresonator biosensors with sensitivity.

\section{EXPERIMENTAL DESIGN}

We used microtoroids because they have superior optical properties regarding sensing (4), they can be fabricated on chip, and our research group had previously reported lasing action from polymer coated microtoroids (5). We fabricated silica microdisks via standard photolithography and dry etch processes. A subsequent $\mathrm{CO}_{2}$ laser exposure forms uniform microtoroids, which are inspected with SEM. Light from a tunable external cavity laser was coupled inside the microcavity by a tapered fiber, fabricated by $\mathrm{CO}_{2}$ laser heating and pulling, and subsequent HF treatment. The output was collected by a photodetector. Piezo actuators were used for precise positioning, and the whispering gallery modes were investigated. We used thiol containing self assembly monolayers (SAMs) with two different terminal ends (-OH and $-\mathrm{COOH}$ ) in order to attain selectivity. Microtoroids were coated with $1 \mathrm{~nm} \mathrm{Au-Pd,} \mathrm{on} \mathrm{which} \mathrm{SAMs} \mathrm{readily} \mathrm{assemble} \mathrm{by}$ covalent interactions. The carboxyl end of SAM was covalently attached to antibodies against Escherichia Coli via EDC/NHS reaction. Each modification was analyzed by XPS and ellipsometry measurements, and whispering gallery modes were observed after each step individually. E. Coli and were grown in LB media with ampicillin.

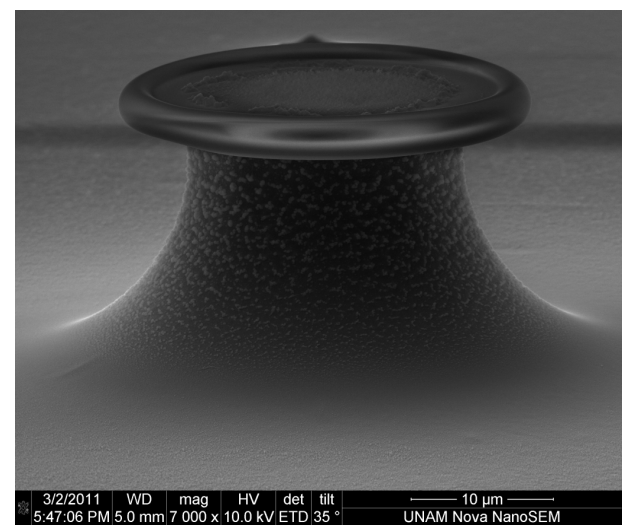

Figure 1: SEM micrograph of the toroidal microcavity.

\section{RESULTS}

The whispering gallery modes were observed to be dependent on the diameter of the microcavity. We estimated the quality factor of the microtoroids from the relation:

$$
Q=\frac{\lambda}{\Delta \lambda}
$$


Among microtoroids having diameters $60-120 \mathrm{~mm}$, we used the ones with highest quality factors. After the surface modification of the microtoroids, we tested different concentrations of $E$. Coli in PBS buffer, and observed single bacterium binding events. We measured presence of $E$. Coli in serum, and found comparable results, showing the selectivity in our system.
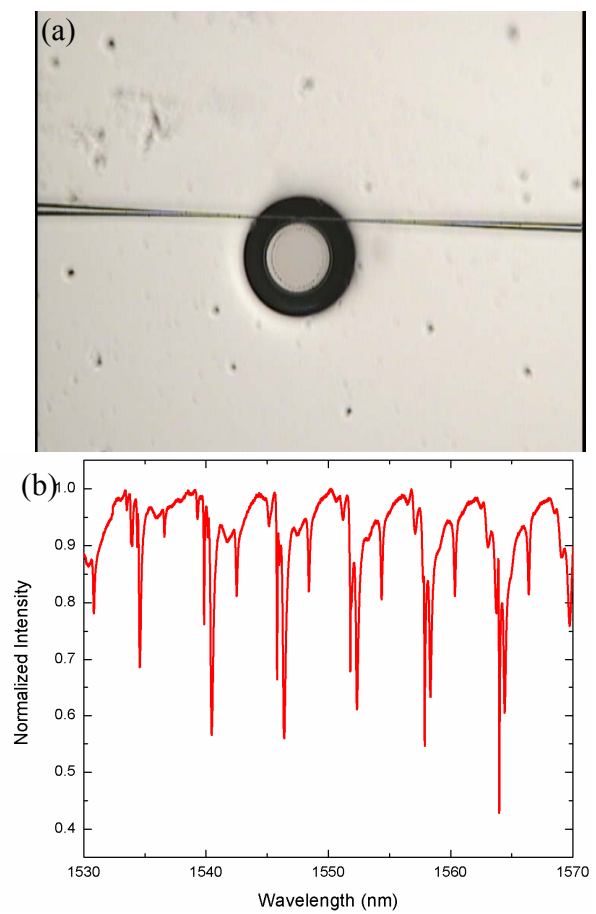

Figure 2: (a) Coupling of light inside optical microcavity. (b) Observed whispering gallery modes of the toroidal microcavity.

\section{DISCUSSION}

SAMs are used in this research because they provide a facile method for surface applications. The surface modification strategy utilized in this work, which is quite robust and reliable, can be extended broadly in order to detect any type of biological and chemical species. This would be important in using microcavity biosensors in daily life applications. Future directions in this work would be utilization of different strategies for selectively detecting different types of species. One proposed strategy is the use of aptamers instead of antibodies, because they possess higher sensitivity towards their targets, and flexibility in their design. Another current aim of our research group is to extend the fabrication of microtoroids in order to have all system on-chip assembled. Therefore, commercialization of microcavity based sensors would be more feasible.

\section{REFERENCES}

[1] F. Vollmer and S. Arnold, "Whispering-gallery-mode biosensing: labelfree detection down to single molecules," Nature Methods, vol. 5, pp 591-596, Jul 2008.

[2] A. M. Armani, R. P. Kulkarni, S. E. Fraser, R. C. Flagan, and K. J. Vahala, "Label-free, single-molecule detection with optical microcavities," Science, vol. 317, pp. 783-787, Aug 102007.

[3] K. De Vos, J. Girones, T. Claes, Y. De Koninck, S. Popelka, E. Schacht, R. Baets, and P. Bienstman, "Multiplexed Antibody Detection With an Array of Silicon-on-Insulator Microring Resonators," Ieee Photonics Journal, vol. 1, pp. 225-235, Oct 2009.

[4] K. J. Vahala, "Optical microcavities," Nature, vol. 424, pp. 839-846, Aug 142003.

[5] A. Tulek, D. Akbulut, and M. Bayindir, "Ultralow threshold laser action from toroidal polymer microcavity," Applied Physics Letters, vol. 94, pp. 203302-4, May 182009. 